\title{
De novo design of picomolar SARS-CoV-2 miniprotein inhibitors
}

\begin{abstract}
Longxing Cao ${ }^{1,2}$, Inna Goreshnik ${ }^{1,2}$, Brian Coventry ${ }^{1,2,3}$, James Brett Case ${ }^{4}$, Lauren Miller $^{1,2}$, Lisa Kozodoy ${ }^{1,2}$, Rita E. Chen $^{4,5}$, Lauren Carter $^{1,2}$,

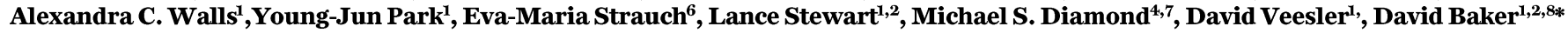
1Department of Biochemistry, University of Washington, Seattle, WA 98195, USA. Institute for Protein Design, University of Washington, Seattle, WA 98195, USA. ${ }^{3}$ Molecular Engineering Graduate Program, University of Washington, Seattle, WA 98195, USA. ${ }^{4}$ Department of Medicine, Washington University School of Medicine, St. Louis, MO 63110, USA. 5Department of Pathology and Immunology, Washington University School of Medicine, St. Louis, MO 63110, USA. ${ }^{6}$ Department of Pharmaceutical and Biomedical Sciences, University of Georgia, Athens, GA 30602, USA. TThe Andrew M. and Jane M. Bursky Center for Human Immunology and Immunotherapy Programs, Washington University School of Medicine, St. Louis, MO 63110, USA. ${ }^{8 H}$ Howard Hughes Medical Institute, University of Washington, Seattle, WA 98195, USA.

*Corresponding author. E-mail: dabaker@uw.edu

Targeting the interaction between the SARS-CoV-2 Spike protein and the human ACE2 receptor is a promising therapeutic strategy. We designed inhibitors using two de novo design approaches. Computer generated scaffolds were either built around an ACE2 helix that interacts with the Spike receptor binding domain (RBD), or docked against the RBD to identify new binding modes, and their amino acid sequences designed to optimize target binding, folding and stability. Ten designs bound the RBD with affinities ranging from 100pM to 10nM, and blocked ARS-CoV-2 infection of Vero E6 cells with IC 50 values between 24 pM and $35 \mathrm{nM}$; The most potent, with new binding modes, are 56 and 64 residue proteins (IC $50 \sim 0.16 \mathrm{ng} / \mathrm{ml}$ ). Cryo-electron microscopy structures of these minibinders in complex with the SARS-CoV-2 spike ectodomain trimer with all three RBDs bound are nearly identical to the computational models. These hyperstable minibinders provide starting points for SARS-CoV-2 therapeutics.
\end{abstract}

SARS-CoV-2 infection generally begins in the nasal cavity, with virus replicating there for several days before spreading to the lower respiratory tract (1). Delivery of a high concentration of a viral inhibitor into the nose and into the respiratory system generally might therefore provide prophylactic protection and/or therapeutic benefit for treatment of early infection, and could be particularly useful for healthcare workers and others coming into frequent contact with infected individuals. A number of monoclonal antibodies are in development as systemic treatments for COVID-19 (2-6), but these proteins are not ideal for intranasal delivery as antibodies are large and often not extremely stable molecules and the density of binding sites is low (two per $150 \mathrm{KDa}$. antibody); antibody-dependent disease enhancement (7-9) is also a potential issue. High-affinity Spike protein binders that block the interaction with the human cellular receptor angiotensinconverting enzyme 2 (ACE2) (10) with enhanced stability and smaller sizes to maximize the density of inhibitory domains could have advantages over antibodies for direct delivery into the respiratory system through intranasal administration, nebulization or dry powder aerosol. We found previously that intranasal delivery of small proteins designed to bind tightly to the influenza hemagglutinin can provide both prophylactic and therapeutic protection in rodent models of lethal influenza infection (11).

\section{Design strategy}

We set out to design high-affinity protein minibinders to the SARS-CoV-2 Spike RBD that compete with ACE2 binding. We explored two strategies: first we incorporated the alpha-helix from ACE2 which makes the majority of the interactions with the RBD into small designed proteins that make additional interactions with the RBD to attain higher affinity (Fig. 1A). Second, we designed binders completely from scratch without relying on known RBD-binding interactions (Fig. 1B). An advantage of the second approach is that the range of possibilities for design is much larger, and so potentially a greater diversity of high-affinity binding modes can be identified. For the first approach, we used the Rosetta blueprint builder to generate miniproteins which incorporate the ACE2 helix (human ACE2 residues 23 to 46). For the second approach, we used RIF docking (12) and design using large miniprotein libraries (11) to generate binders to distinct regions of the RBD surface surrounding the ACE2 binding site (Fig. 1 and fig. S1).

\section{Experimental characterization and optimization}

Large pools of designed minibinders (see Methods) made using the first and second approaches, were encoded in long oligonucleotides and screened for binding to fluorescently tagged RBD displayed on the surface of yeast cells. Deep 
sequencing identified three ACE2 helix scaffolded designs (Approach 1), and 105 de novo interface designs (Approach 2) that were enriched following fluorescence activated cell sorting (FACS) for RBD binding. All three ACE2-scaffolded designs and twelve of the de novo designs were expressed in $E$. coli and purified. One of the ACE2-scaffolded designs and eleven of the twelve de novo designs were soluble and bound RBD with affinities ranging from $100 \mathrm{nM}$ to $2 \mathrm{uM}$ in biolayer interferometry (BLI) experiments (figs. S2, A, C, and E, and S3). Affinity maturation of the ACE2-scaffolded design by PCR mutagenesis led to a variant, AHB1, which bound RBD with an affinity of $\sim 1 \mathrm{nM}$ (fig. S4) and blocked binding of ACE2 to the RBD (fig. S5A), consistent with the design model, but had low thermostability (fig. S4C). We generated ten additional designs incorporating the binding helix hairpin of AHB1, and found that one bound the RBD and was thermostable (fig. S2, B, D, and F).

For 50 of the minibinders made using approach 2 and the second generation ACE2 helix scaffolded design, we generated site saturation mutagenesis libraries (SSMs) in which every residue in each design was substituted with each of the 20 amino acids one at a time. Deep sequencing before and after FACS sorting for RBD binding revealed that residues at the binding interface and protein core were largely conserved for 40 out of the 50 Approach 2 minibinders and for the ACE2 helix scaffolded design (Fig. 2 and figs. S6 and S7). For most of these minibinders, a small number of substitutions were enriched in the FACS sorting; combinatorial libraries incorporating these substitutions were constructed for the ACE2based design and the eight highest affinity Approach 2 designs and again screened for binding to the RBD at concentrations down to 20pM. Each library converged on a small number of closely related sequences; one of these was selected for each design AHB2 or LCB1-LCB8 and found to bind the RBD with high affinity on the yeast surface in a manner competed by ACE2 (Fig. 3 and fig. S8).

AHB2 and LCB1-LCB8 were expressed, purified from $E$. coli, and binding to the RBD assessed by BLI. For seven of the designs, the $K_{D}$ values ranged from 1-20 nM (Fig. 3, fig. S8, and table S2), and for two (LCB1 and LCB3), the $\mathrm{K}_{\mathrm{D}}$ values were below $1 \mathrm{nM}$, which is too strong to measure reliably with this technique (Fig. 3). On the surface of yeast cells, LCB1 and LCB3 showed binding signals at $5 \mathrm{pM}$ of RBD following protease (trypsin and chymotrypsin) treatment (fig. S9). Circular dichroism spectra of the purified minibinders were consistent with the design models, and the melting temperatures for most were greater than $90^{\circ} \mathrm{C}$ (Fig. 3 and fig. S8). The designs retained full binding activity after 14 days at room temperature (fi g.S10). AHB1/2 and LCB3 also bound to the SARSCoV RBD (in addition to the SARS-CoV-2 RBD), but with lower affinity (fig. S11); we anticipate the binding affinities achieved for SARS-CoV-2 could be readily obtained for other coronavirus spike proteins if these were directly targeted for design.

\section{CryoEM structure determination}

We characterized the structures of LCB1 and LCB3 in complex with the SARS-CoV-2 spike ectodomain trimer at $2.7 \AA$ and $3.1 \AA$ resolution, respectively, and found that the minibinders bind stoichiometrically to the three RBDs within the spike trimer (Fig. 4, A and E, and figs. S12 and S13). Although the spike predominantly harbored two open RBDs for both complexes, we identified a subset of particles with three RBDs open for the LCB3 complex (Fig. 4, A and E, and figs. S12 and S13). We improved the resolvability of the RBD/LCB1 and RBD/LCB3 densities using focused classification and local refinement yielding maps at 3.1 and $3.5 \AA$ resolution enabling visualization of the interactions formed by each minibinder with the RBD (Fig. 4, B and F, and figs. S12 and S13).

LCB1 and LCB3 dock with opposite orientations in the crevice formed by the RBD receptor-binding motif through extensive shape complementary interfaces with numerous electrostatic interactions mediated by two out of the three minibinder $\alpha$-helices (Fig. 4, B to D and F to H). Similar to ACE2, the LCB1 and LCB3 binding sites are buried in the closed S conformational state and require opening of at least two RBDs to allow simultaneous recognition of the three binding sites (Fig. 4, A and E). Both LCB1 and LCB3 form multiple hydrogen bonds and salt bridges with the RBD with buried surface areas of $\sim 1,000 \AA^{\wedge} 2$ and $\sim 800 \AA^{\wedge} 2$, respectively (Fig. 4, C, D, G, and H), consistent with the subnanomolar affinities of these inhibitors. As designed, the binding sites for LCB1 and LCB3 overlap with that of ACE2 (fig. S14 and table S1), and hence should compete for binding to the RBD and inhibit viral attachment to the host cell surface.

Superimposition of the designed LCB1/RBD or LCB3/RBD models to the corresponding cryoEM structures, using the RBD as reference, show that the binding poses closely match the design with backbone Ca rmsd of $1.27 \AA$ and $1.9 \AA \AA$ for LCB1 and LCB3, respectively (Fig. 4, B and F) and most of the polar interactions in the design models closely match the CryoEM structure (Fig. 4, C, D, G, and H). These data show that the computational design method can have quite high accuracy. The structure comparisons in Fig. 4, C, D, G, and H, are to the original design models; the substitutions that increased binding affinity are quite subtle and have very little effect on backbone geometry.

\section{Virus neutralization}

We investigated the capacity of AHB1, AHB2 and LCB1-5 to prevent infection of cells by bona fide SARS-CoV-2. Varying concentrations of minibinders were incubated with 100 focus-forming units (FFU) of SARS-CoV-2 and then added to Vero E6 monolayers. AHB1 and AHB2 strongly neutralized 
SARS-CoV-2 ( $\mathrm{IC}_{50}$ of $35 \mathrm{nM}$ and $15.5 \mathrm{nM}$ respectively), whereas a control influenza minibinder showed no neutralization activity (Fig. 5A). Next, we tested the Approach 2 designed minibinders LCB1-5. We observed even more potent neutralization of SARS-CoV-2 by LCB1 and LCB3 with $\mathrm{IC}_{50}$ values of $23.54 \mathrm{pM}$ and $48.1 \mathrm{pM}$, respectively (Fig. $5 \mathrm{~B}$; at increased incubation volumes, IC50's as low as $11 \mathrm{pM}$ were obtained). On a molar basis, these values are approximately 3fold lower than the most potent anti-SARS-CoV-2 monoclonal antibody described to date (13); on a mass basis, because of their very small size, the designs are even more potent than the antibodies.

\section{Conclusions}

The minibinders designed in this work have potential advantages over antibodies as potential therapeutics. Together, they span a range of binding modes, and in combination viral mutational escape would be quite unlikely (figs. S1 and S14 and table S1). The retention of activity after extended time at elevated temperatures suggests they would not require a temperature-controlled supply chain. The designs are 20-fold smaller than a full antibody molecule, and hence in an equal mass have 20 -fold more potential neutralizing sites, increasing the potential efficacy of a locally administered drug. The cost of goods and the ability to scale to very high production should be lower for the much simpler miniproteins, which unlike antibodies, do not require expression in mammalian cells for proper folding. The small size and high stability should also make them amenable to formulation in a gel for nasal application, and to direct delivery into the respiratory system by nebulization or as a dry powder. We will be exploring alternative routes of delivery in the months ahead as we seek to translate the high potency neutralizing proteins into SARS-Cov2 therapeutics and prophylactics. Immunogenicity is a potential problem with any foreign molecule, but for previously characterized small de novo designed proteins little or no immune response has been observed $(11,14)$, perhaps because the high solubility and stability together with the small size makes presentation on dendritic cells less likely.

Timing is critical in a pandemic outbreak: potent therapeutics are needed in as short a time as possible. We began to design minibinders in January 2020 based on a Rosetta model of the SARS-CoV-2 Spike structure and switched to the crystal structures once they became available $(4,15-17)$. By the end of May 2020, we had identified very potent neutralizers of infectious virus; during this same time, a number of neutralizing monoclonal antibodies were identified. We believe that with continued development, the computational design approach can become much faster. First, as structure prediction methods continue to increase in accuracy, target models suitable for design could be generated within a day of determining the genome sequence of a new pathogen.
Second, with continued improvement in computational design methods, it should be possible to streamline the workflow described here, which required screening of large sets of computational designs, followed by experimental optimization, to identify very high affinity binders. The very close agreement of the cryoEM structures of LCB1 and LCB3 with the computational design models suggest that the main challenges to overcome are not in the de novo design of proteins with shape and chemical complementarity to the target surface, but in recognizing the best candidates and identifying a small number of affinity increasing substitutions. The large amount of data collected in protein interface design experiments such as those described here should inform the improvement of the detailed atomic models at the core of Rosetta design calculations, as well as complementary machine learning approaches, to enable recognition and improved sequence design of the best candidates; this would enable even faster in silico design of pM inhibitors like LCB1 and LCB3. With continued methods development, we believe that it will become possible to generate ultra-high-affinity, pathogen neutralizing designs within weeks of obtaining genome sequence. Preparing against unknown future pandemics is difficult, and such a capability could be an important component of a general response strategy.

\section{REFERENCES AND NOTES}

1. Y. J. Hou, K. Okuda, C. E. Edwards, D. R. Martinez, T. Asakura, K. H. Dinnon 3rd, T. Kato, R. E. Lee, B. L. Yount, T. M. Mascenik, G. Chen, K. N. Olivier, A. Ghio, L. V. Tse, S. R. Leist, L. E. Gralinski, A. Schäfer, H. Dang, R. Gilmore, S. Nakano, L. Sun, M. L. Fulcher, A. Livraghi-Butrico, N. I. Nicely, M. Cameron, C. Cameron, D. J. Kelvin, A. de Silva, D. M. Margolis, A. Markmann, L. Bartelt, R. Zumwalt, F. J. Martinez, S. P. Salvatore, A. Borczuk, P. R. Tata, V. Sontake, A. Kimple, I. Jaspers, W. K. O'Neal, S. H. Randell, R. C. Boucher, R. S. Baric, SARS-CoV-2 Reverse Genetics Reveals a Variable Infection Gradient in the Respiratory Tract. Cell 182, 429-446.e14 (2020). doi:10.1016/i.cell.2020.05.042 Medline

2. R. Shi, C. Shan, X. Duan, Z. Chen, P. Liu, J. Song, T. Song, X. Bi, C. Han, L. Wu, G. Gao, X. Hu, Y. Zhang, Z. Tong, W. Huang, W. J. Liu, G. Wu, B. Zhang, L. Wang, J. Qi, H. Feng, F. S. Wang, Q. Wang, G. F. Gao, Z. Yuan, J. Yan, A human neutralizing antibody targets the receptor-binding site of SARS-CoV-2. Nature 584, 120-124 (2020). doi:10.1038/s41586-020-2381-y Medline

3. D. Pinto, Y.-J. Park, M. Beltramello, A. C. Walls, M. A. Tortorici, S. Bianchi, S. Jaconi, K. Culap, F. Zatta, A. De Marco, A. Peter, B. Guarino, R. Spreafico, E. Cameroni, J. B. Case, R. E. Chen, C. Havenar-Daughton, G. Snell, A. Telenti, H. W. Virgin, A. Lanzavecchia, M. S. Diamond, K. Fink, D. Veesler, D. Corti, Structural and functional analysis of a potent sarbecovirus neutralizing antibody. bioRxiv 023903 [Preprint] 10 April 2020; https://doi.org/10.1101/2020.04.07.023903.

4. J. Lan, J. Ge, J. Yu, S. Shan, H. Zhou, S. Fan, Q. Zhang, X. Shi, Q. Wang, L. Zhang, X. Wang, Structure of the SARS-CoV-2 spike receptor-binding domain bound to the ACE2 receptor. Nature 581, 215-220 (2020). doi:10.1038/s41586-020-2180-5 Medline

5. M. Yuan, N. C. Wu, X. Zhu, C. D. Lee, R. T. Y. So, H. Lv, C. K. P. Mok, I. A. Wilson, A highly conserved cryptic epitope in the receptor binding domains of SARS-CoV-2 and SARS-CoV. Science 368, 630-633 (2020). doi:10.1126/science.abb7269 Medline

6. Y. Wu, F. Wang, C. Shen, W. Peng, D. Li, C. Zhao, Z. Li, S. Li, Y. Bi, Y. Yang, Y. Gong, H. Xiao, Z. Fan, S. Tan, G. Wu, W. Tan, X. Lu, C. Fan, Q. Wang, Y. Liu, C. Zhang, J. 
Qi, G. F. Gao, F. Gao, L. Liu, A noncompeting pair of human neutralizing antibodies block COVID-19 virus binding to its receptor ACE2. Science 368, 1274-1278 (2020). doi:10.1126/science.abc2241 Medline

7. K. L. Winarski, J. Tang, L. Klenow, J. Lee, E. M. Coyle, J. Manischewitz, H. L. Turner, K. Takeda, A. B. Ward, H. Golding, S. Khurana, Antibody-dependent enhancement of influenza disease promoted by increase in hemagglutinin stem flexibility and virus fusion kinetics. Proc. Natl. Acad. Sci. U.S.A. 116, 15194-15199 (2019). doi:10.1073/pnas.1821317116 Medline

8. A. Taylor, S.-S. Foo, R. Bruzzone, L. V. Dinh, N. J. C. King, S. Mahalingam, Fo receptors in antibody-dependent enhancement of viral infections. Immunol. Rev. 268, 340-364 (2015). doi:10.1111/imr.12367 Medline

9. B. S. Graham, Rapid COVID-19 vaccine development. Science 368, 945-946 (2020). doi:10.1126/science abb8923 Medline

10. A. C. Walls, Y.-J. Park, M. A. Tortorici, A. Wall, A. T. McGuire, D. Veesler, Structure, function and antigenicity of the SARS-CoV-2 spike glycoprotein. bioRxiv 956581 [Preprint] 20 February 2020. https://doi.org/10.1101/2020.02.19.956581.

11. A. Chevalier, D. A. Silva, G. J. Rocklin, D. R. Hicks, R. Vergara, P. Murapa, S. M. Bernard, L. Zhang, K. H. Lam, G. Yao, C. D. Bahl, S. I. Miyashita, I. Goreshnik, J. T. Fuller, M. T. Koday, C. M. Jenkins, T. Colvin, L. Carter, A. Bohn, C. M. Bryan, D. A. Fernández-Velasco, L. Stewart, M. Dong, X. Huang, R. Jin, I. A. Wilson, D. H. Fuller, D. Baker, Massively parallel de novo protein design for targeted therapeutics. Nature 550, 74-79 (2017). doi:10.1038/nature23912 Medline

12. J. Dou, A. A. Vorobieva, W. Sheffler, L. A. Doyle, H. Park, M. J. Bick, B. Mao, G. W. Foight, M. Y. Lee, L. A. Gagnon, L. Carter, B. Sankaran, S. Ovchinnikov, E. Marcos, P. S. Huang, J. C. Vaughan, B. L. Stoddard, D. Baker, De novo design of a fluorescence-activating $\beta$-barrel. Nature 561, 485-491 (2018). doi:10.1038/s41586-018-0509-0 Medline

13. W. B. Alsoussi, J. S. Turner, J. B. Case, H. Zhao, A. J. Schmitz, J. Q. Zhou, R. E. Chen, T. Lei, A. A. Rizk, K. M. McIntire, E. S. Winkler, J. M. Fox, N. M. Kafai, L. B. Thackray, A. O. Hassan, F. Amanat, F. Krammer, C. T. Watson, S. H. Kleinstein, D. H. Fremont, M. S. Diamond, A. H. Ellebedy, A Potently Neutralizing Antibody Protects Mice against SARS-CoV-2 Infection. J. Immunol. 205, 915-922 (2020). doi:10.4049/iimmunol.2000583 Medline

14. D. A. Silva, S. Yu, U. Y. Ulge, J. B. Spangler, K. M. Jude, C. Labão-Almeida, L. R. Ali, A. Quijano-Rubio, M. Ruterbusch, I. Leung, T. Biary, S. J. Crowley, E. Marcos, C. D. Walkey, B. D. Weitzner, F. Pardo-Avila, J. Castellanos, L. Carter, L. Stewart, S. R. Riddell, M. Pepper, G. J. L. Bernardes, M. Dougan, K. C. Garcia, D. Baker, De novo design of potent and selective mimics of IL-2 and IL-15. Nature 565, 186-191 (2019). doi:10.1038/s41586-018-0830-7 Medline

15. J. Shang, G. Ye, K. Shi, Y. Wan, C. Luo, H. Aihara, Q. Geng, A. Auerbach, F. Li, Structural basis of receptor recognition by SARS-CoV-2. Nature 581, 221-224 (2020). doi:10.1038/s41586-020-2179-y Medline

16. Q. Wang, Y. Zhang, L. Wu, S. Niu, C. Song, Z. Zhang, G. Lu, C. Qiao, Y. Hu, K. Y. Yuen, Q. Wang, H. Zhou, J. Yan, J. Qi, Structural and Functional Basis of SARS-CoV-2 Entry by Using Human ACE2. Cell 181, 894-904.e9 (2020). doi:10.1016/i.cell.2020.03.045 Medline

17. R. Yan, Y. Zhang, Y. Li, L. Xia, Y. Guo, Q. Zhou, Structural basis for the recognition of SARS-CoV-2 by full-length human ACE2. Science 367, 1444-1448 (2020). doi:10.1126/science. abb2762 Medline

18. P. S. Huang, Y.-E. A. Ban, F. Richter, I. Andre, R. Vernon, W. R. Schief, D. Baker, RosettaRemodel: A generalized framework for flexible backbone protein design. PLOS ONE 6, e24109 (2011). doi:10.1371/journal.pone.0024109 Medline

19. N. Koga, R. Tatsumi-Koga, G. Liu, R. Xiao, T. B. Acton, G. T. Montelione, D. Baker, Principles for designing ideal protein structures. Nature 491, 222-227 (2012). doi:10.1038/nature11600 Medline

20. D. M. Hoover, J. Lubkowski, DNAWorks: An automated method for designing oligonucleotides for PCR-based gene synthesis. Nucleic Acids Res. 30, e43 (2002). doi:10.1093/nar/30.10.e43 Medline

21. C. L. Hsieh, J. A. Goldsmith, J. M. Schaub, A. M. DiVenere, H. C. Kuo, K. Javanmardi, K. C. Le, D. Wrapp, A. G. Lee, Y. Liu, C. W. Chou, P. O. Byrne, C. K. Hjorth, N. V. Johnson, J. Ludes-Meyers, A. W. Nguyen, J. Park, N. Wang, D. Amengor, J. A. Maynard, I. J. Finkelstein, J. S. McLellan, Structure-based Design of Prefusionstabilized SARS-CoV-2 Spikes. bioRxiv 2020.05.30.125484 (2020). https://doi.org/10.1101/2020.05.30.125484 Medline
22. C. Suloway, J. Pulokas, D. Fellmann, A. Cheng, F. Guerra, J. Quispe, S. Stagg, C. S. Potter, B. Carragher, Automated molecular microscopy: The new Leginon system. J. Struct. Biol. 151, 41-60 (2005). doi:10.1016/i.jsb.2005.03.010 Medline

23. D. Tegunov, P. Cramer, Real-time cryo-electron microscopy data preprocessing with Warp. Nat. Methods 16, 1146-1152 (2019). doi:10.1038/s41592-019-0580-y Medline

24. A. Punjani, J. L. Rubinstein, D. J. Fleet, M. A. Brubaker, cryoSPARC: Algorithms for rapid unsupervised cryo-EM structure determination. Nat. Methods 14, 290-296 (2017). doi:10.1038/nmeth.4169 Medline

25. D. Kimanius, B. O. Forsberg, S. H. Scheres, E. Lindahl, Accelerated cryo-EM structure determination with parallelisation using GPUs in RELION-2. eLife 5, e18722 (2016). doi:10.7554/eLife.18722 Medline

26. J. Zivanov, T. Nakane, B. O. Forsberg, D. Kimanius, W. J. H. Hagen, E. Lindahl, S. H. W. Scheres, New tools for automated high-resolution cryo-EM structure determination in RELION-3. elife 7, e42166 (2018). doi:10.7554/eLife.42166 Medline

27. A. Punjani, H. Zhang, D. J. Fleet, Non-uniform refinement: Adaptive regularization improves single particle cryo-EM reconstruction. bioRxiv 877092 [Preprint] 16 December 2019. https://doi.org/10.1101/2019.12.15.877092.

28. J. Zivanov, T. Nakane, S. H. W. Scheres, A Bayesian approach to beam-induced motion correction in cryo-EM single-particle analysis. IUCrJ 6, 5-17 (2019) doi:10.1107/S205225251801463X Medline

29. S. Chen, G. McMullan, A. R. Faruqi, G. N. Murshudov, J. M. Short, S. H. W. Scheres, R. Henderson, High-resolution noise substitution to measure overfitting and validate resolution in 3D structure determination by single particle electron cryomicroscopy. Ultramicroscopy 135, 24-35 (2013) do:10.1016/i.ultramic.2013.06.004 Medline

30. E. F. Pettersen, T. D. Goddard, C. C. Huang, G. S. Couch, D. M. Greenblatt, E. C. Meng, T. E. Ferrin, UCSF Chimera-A visualization system for exploratory research and analysis. J. Comput. Chem. 25, 1605-1612 (2004). doi:10.1002/icc.20084 Medline

31. A. Casañal, B. Lohkamp, P. Emsley, Current developments in Coot for macromolecular model building of Electron Cryo-microscopy and Crystallographic Data. Protein Sci. 29, 1069-1078 (2020). doi:10.1002/pro.3791 Medline

32. B. Frenz, S. Rämisch, A. J. Borst, A. C. Walls, J. Adolf-Bryfogle, W. R. Schief, D. Veesler, F. DiMaio, Automatically fixing errors in glycoprotein structures with Rosetta. Structure 27, 134-139.e3 (2019). doi:10.1016/i.str 2018.09.006 Medline

33. F. DiMaio, Y. Song, X. Li, M. J. Brunner, C. Xu, V. Conticello, E. Egelman, T. Marlovits, Y. Cheng, D. Baker, Atomic-accuracy models from 4.5-Å cryo-electron microscopy data with density-guided iterative local refinement. Nat. Methods 12 , 361-365 (2015). doi:10.1038/nmeth.3286 Medline

34. R. Y. Wang, Y. Song, B. A. Barad, Y. Cheng, J. S. Fraser, F. DiMaio, Automated structure refinement of macromolecular assemblies from cryo-EM maps using Rosetta. elife 5, e17219 (2016). doi:10.7554/elife.17219 Medline

35. C. J. Williams, J. J. Headd, N. W. Moriarty, M. G. Prisant, L. L. Videau, L. N. Deis, V. Verma, D. A. Keedy, B. J. Hintze, V. B. Chen, S. Jain, S. M. Lewis, W. B. Arendall 3rd, J. Snoeyink, P. D. Adams, S. C. Lovell, J. S. Richardson, D. C. Richardson, MolProbity: More and better reference data for improved all-atom structure validation. Protein Sci. 27, 293-315 (2018). doi:10.1002/pro.3330 Medline

36. D. Liebschner, P. V. Afonine, M. L. Baker, G. Bunkóczi, V. B. Chen, T. I. Croll, B. Hintze, L.-W. Hung, S. Jain, A. J. McCoy, N. W. Moriarty, R. D. Oeffner, B. K. Poon, M. G. Prisant, R. J. Read, J. S. Richardson, D. C. Richardson, M. D. Sammito, O. V. Sobolev, D. H. Stockwell, T. C. Terwilliger, A. G. Urzhumtsev, L. L. Videau, C. J. Williams, P. D. Adams, Macromolecular structure determination using X-rays, neutrons and electrons: Recent developments in Phenix. Acta Crystallogr. D Struct. Biol. 75, 861-877 (2019). doi:10.1107/S2059798319011471 Medline

37. J. Agirre, J. Iglesias-Fernández, C. Rovira, G. J. Davies, K. S. Wilson, K. D. Cowtan, Privateer: Software for the conformational validation of carbohydrate structures. Nat. Struct. Mol. Biol. 22, 833-834 (2015). doi:10.1038/nsmb.3115 Medline

38. B. A. Barad, N. Echols, R. Y.-R. Wang, Y. Cheng, F. DiMaio, P. D. Adams, J. S. Fraser, EMRinger: Side chain-directed model and map validation for 3D cryo-electron microscopy. Nat. Methods 12, 943-946 (2015). doi:10.1038/nmeth.3541 Medline 
39. T. D. Goddard, C. C. Huang, E. C. Meng, E. F. Pettersen, G. S. Couch, J. H. Morris, T. E. Ferrin, UCSF ChimeraX: Meeting modern challenges in visualization and analysis. Protein Sci. 27, 14-25 (2018). doi:10.1002/pro.3235 Medline

\section{ACKNOWLEDGMENTS}

We thank Samer Halabiya for MiSeq support, Erik Procko for Fc tagged RBD protein, Kandise Van Wormer and Austin Curtis Smith for their tremendous laboratory support during COVID-19. Funding: This work was supported by DARPA Synergistic Discovery and Design (SD2) HR0011835403 contract FA8750-17-C0219 (L.C., B.C., D.B.), The Audacious Project at the Institute for Protein Design (L.K., L.C.), funding from Eric and Wendy Schmidt by recommendation of the Schmidt Futures program (L.M.,I.G.) the Open Philanthropy Project Improving Protein Design Fund (B.C.,D.B.), an Azure computing resource gift for COVID-19 research provided by Microsoft (L.C.,B.C.), the National Institute of General Medical Sciences (R01GM120553 to D.V.), the National Institute of Allergy and Infectious Diseases (HHSN272201700059C, D.V., D.B., L.S.), a Helen Hay Whitney Foundation postdoctoral fellowship (J.B.C.), a Pew Biomedical Scholars Award (D.V.), an Investigators in the Pathogenesis of Infectious Disease Award from the Burroughs Wellcome Fund (D.V.), a Fast Grant award (D.V.) and the University of Washington Arnold and Mabel Beckman cryo-EM center. Author contribution: L.Cao and D.B. designed the research; L.Cao developed the computational methods for Approach I and made the designs based on the ACE2 helix; L.Cao and B.C. developed the computational methods for Approach II and L.Cao made the de novo designs; B.C., L.Cao and E.M.S. designed the de novo scaffold library; L.Cao, I.G. and L.K. performed the yeast display assays and next generation sequencing; L.Cao, I.G., L.M., L.K., A.C.W. and L. Carter purified and prepared the proteins; L.Cao, I.G. and L.M. performed the BLI assays; L.Cao and L.M. collected the circular dichroism results; Y.P. and D.V. solved the CryoEM structures; J.B.C. and R.E.C. performed the SARS-CoV-2 neutralization assay; L.S., M.S.D., D.V. and D.B. supervised the research; L.Cao, J.B.C., Y.P., L.S., D.V. and D.B wrote the manuscript; all authors discussed the results and commented on the manuscript. Competing interests: L.Cao, I.G., B.C., L.M., L.K., and D.B. are co-inventors on a provisional patent application that incorporates discoveries described in this manuscript. D.B. is a cofounder of Neoleukin Therapeutics. M.S.D. is a consultant for Inbios, Vir Biotechnology, NGM Biopharmaceuticals, and on the Scientific Advisory Board of Moderna. D.V. has a sponsored research agreement from Vir Biotechnology Inc. Data and materials availability: The design models and design scripts used in the manuscript have been deposited to http://files.ipd.uw.edu/pub/SARS-CoV-

2_binder_2020/scripts_models.zip. The cryo-EM maps and atomic models have been deposited at the Electron Microscopy Data Bank and the PDB with accession codes EMD: 22532 and PDB: 7JZL (SARS-CoV-2 S/LCB1), EMD: 22574 and PDB: 7JZU (SARS-CoV-2 S/LCB1, local refinement), EMD: 22534 (SARSCoV-2 S/LCB3, 2 RBDs open), EMD: 22533 and PDB: 7JZM (SARS-CoV-2 S/LCB3, local refinement), EMD: 22535 (SARS-CoV-2 S/LCB3, 3 RBDs open). This work is licensed under a Creative Commons Attribution 4.0 International (CC BY 4.0) license, which permits unrestricted use, distribution, and reproduction in any medium, provided the original work is properly cited. To view a copy of this license, visit https://creativecommons.org/licenses/by/4.0/. This license does not apply to figures/photos/artwork or other content included in the article that is credited to a third party; obtain authorization from the rights holder before using such material.

\section{SUPPLEMENTARY MATERIALS}

science.sciencemag.org/cgi/content/full/science.abd9909/DC1 Materials and Methods

Figs. S1 to S14

Tables S1 to S3

Referencs (18-39)

24 July 2020; accepted 3 September 2020

Published online 9 September 2020

10.1126/science.abd9909 
A

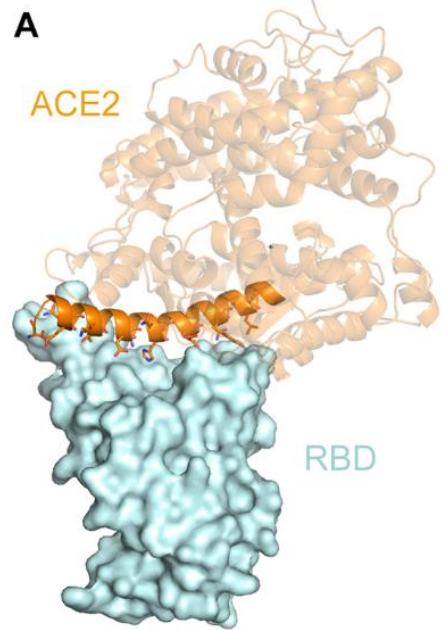

B
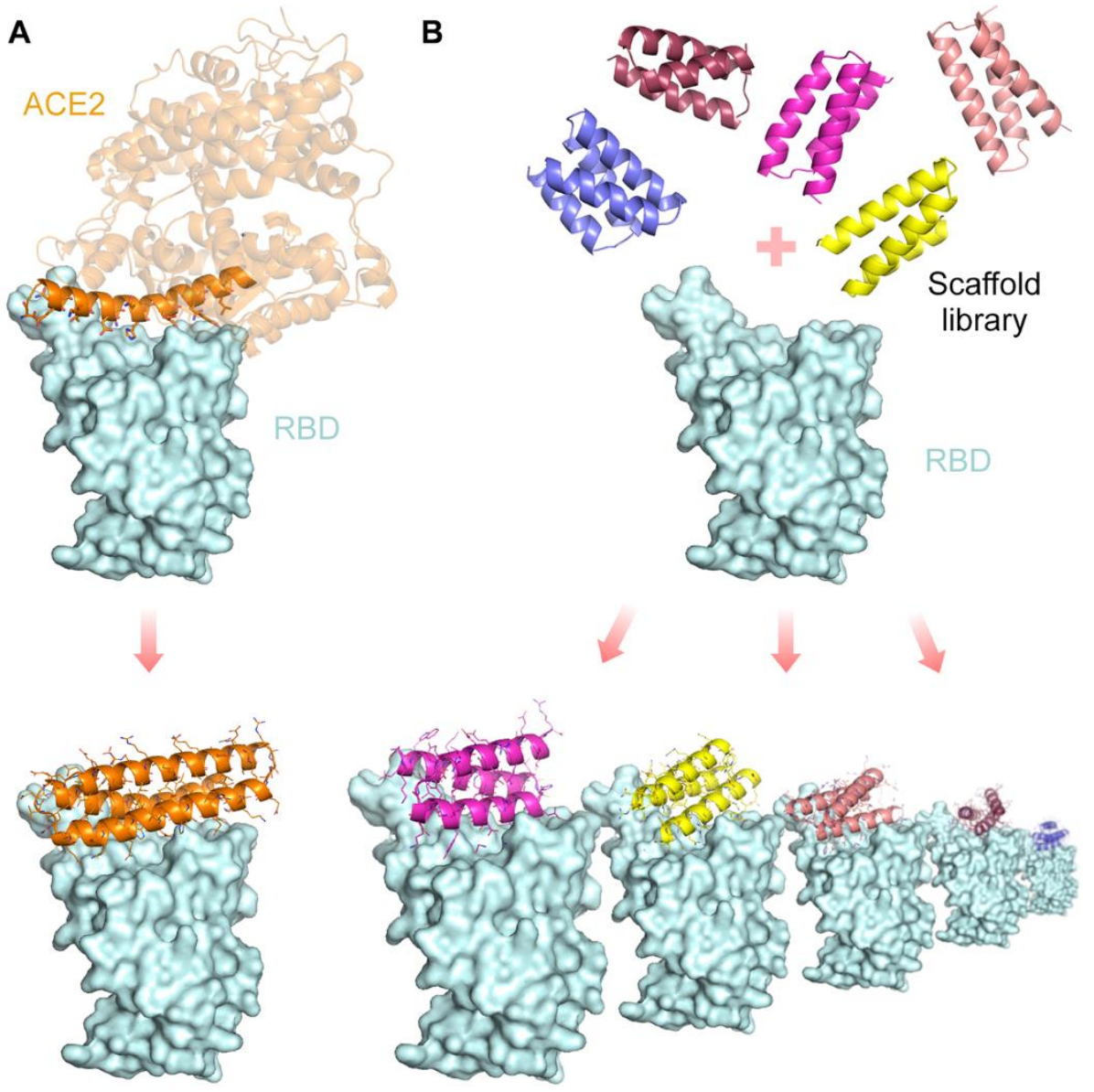

Fig. 1. Overview of the computational design approaches. (A) Design of helical proteins incorporating ACE2 helix. (B) Large scale de novo design of small helical scaffolds (top) followed by rotamer interaction field (RIF) docking to identify shape and chemically complementary binding modes. 
A

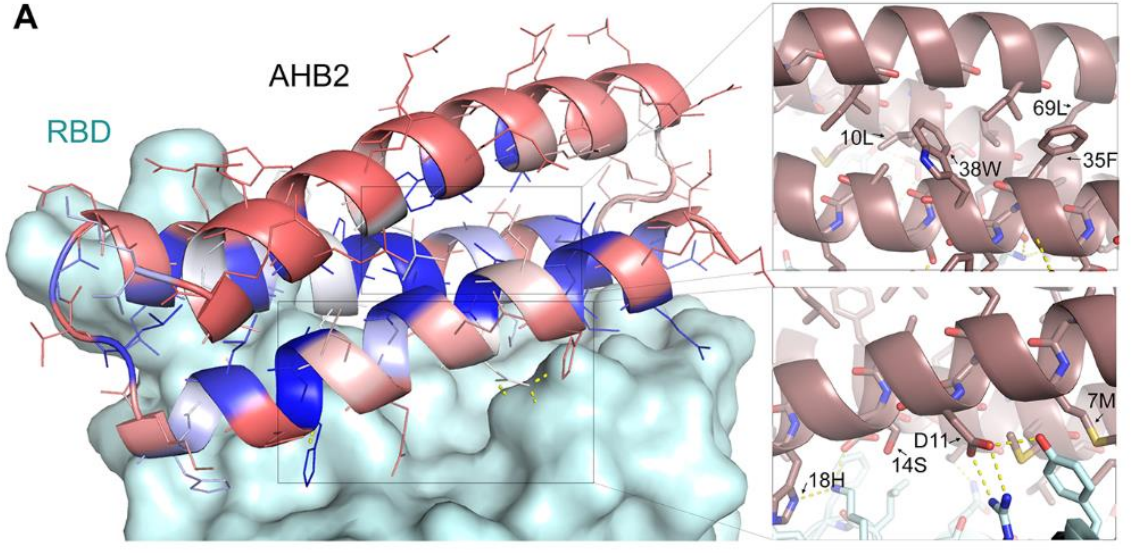

C

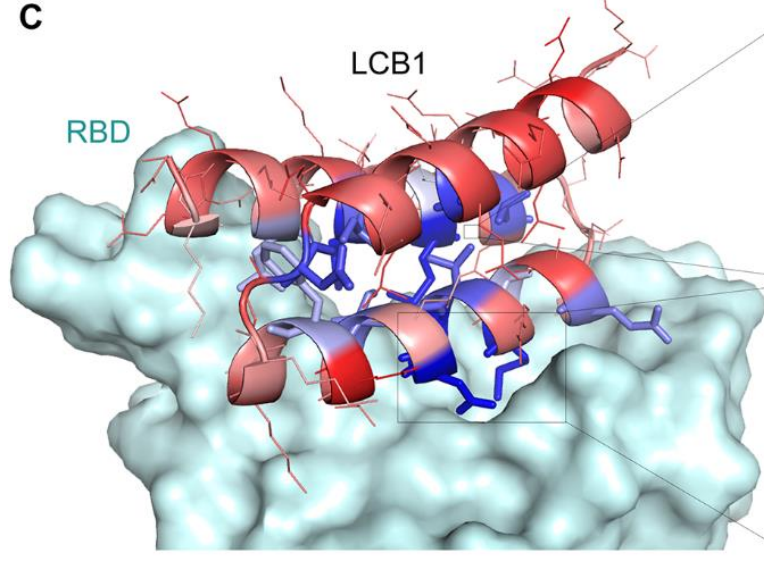

E

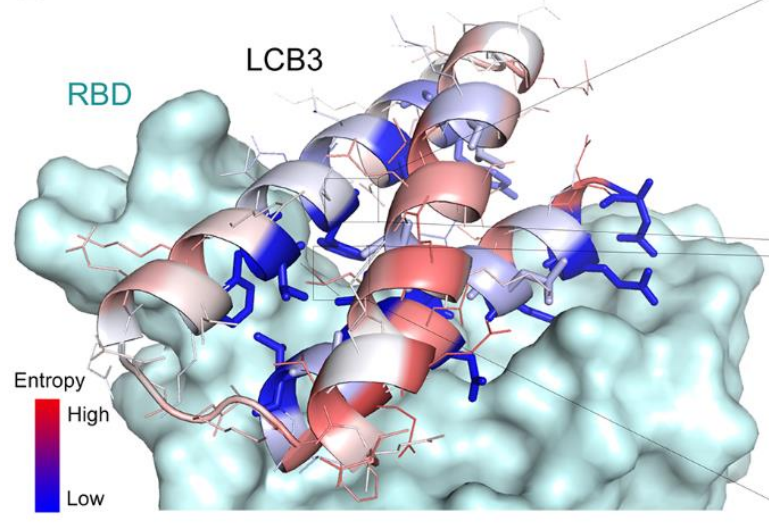

B

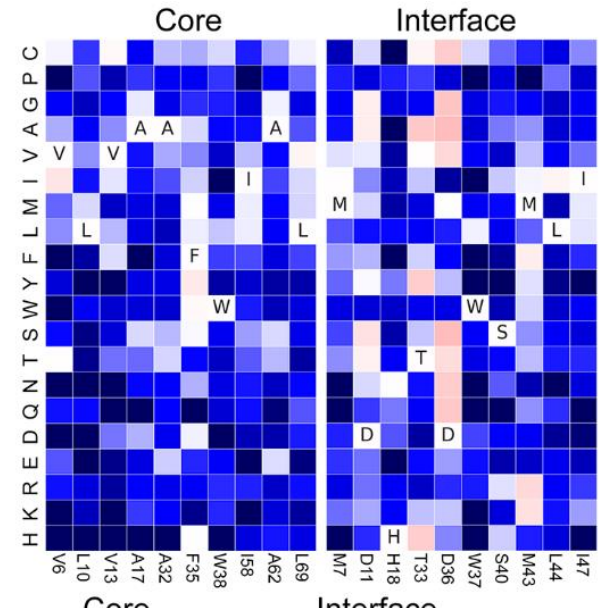

D

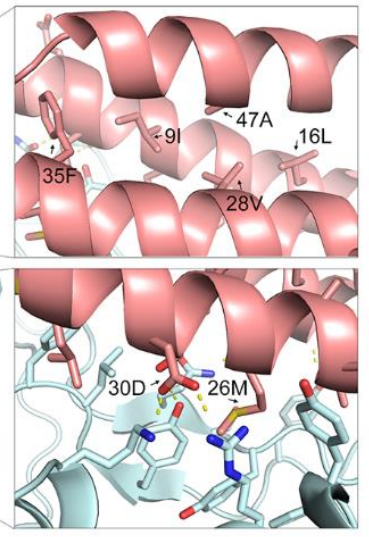

Core

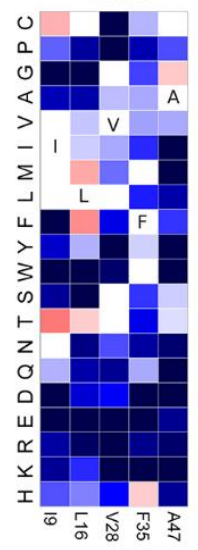

Interface

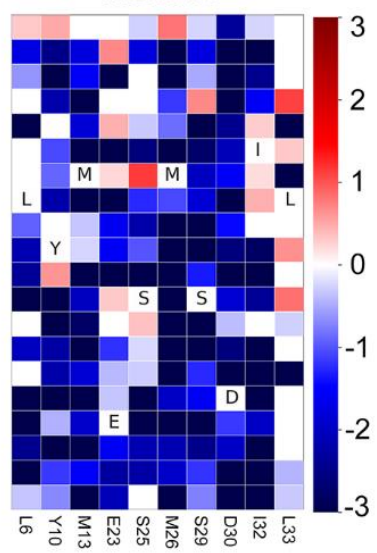

$\mathbf{F}$

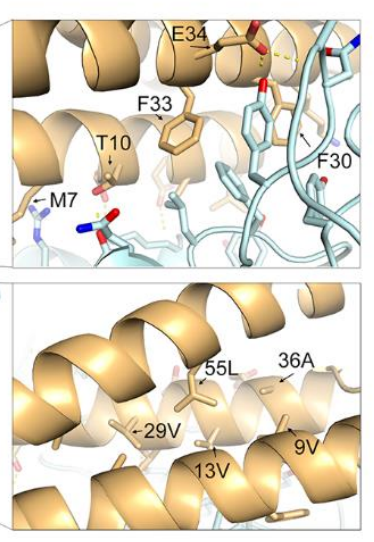

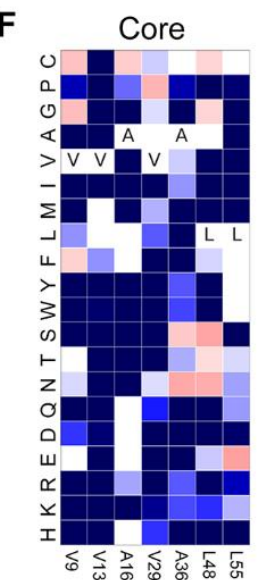

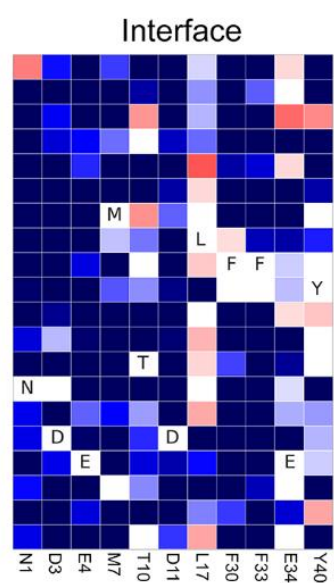

Fig. 2. High resolution sequence mapping of AHB2, LCB1 and LCB3 prior to sequence optimization. (A, C, and E) The designed binding proteins are colored by positional Shannon entropy from site saturation mutagenesis with blue indicating positions of low entropy (conserved) and red those of high entropy (not conserved). (B, D, and F) Heat maps representing RBD-binding enrichment values for single mutations in the design model core (left) and the designed interface (right). Substitutions that are heavily depleted are shown in blue, and beneficial mutations in red. The depletion of most substitutions in both the binding site and the core suggest that the design models are largely correct, while the enriched substitutions suggest routes to improving affinity. Full SSM maps over all positions for AHB2 and all eight de novo designs are provided in figs. S6 and S7. 
A
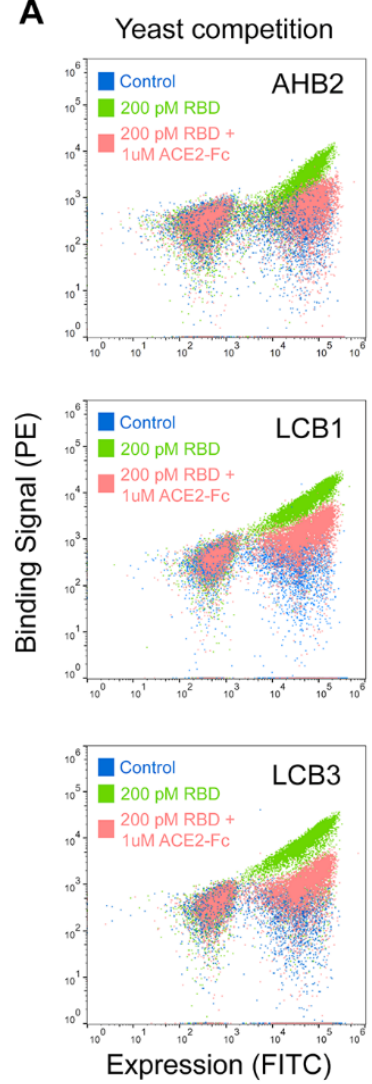

B
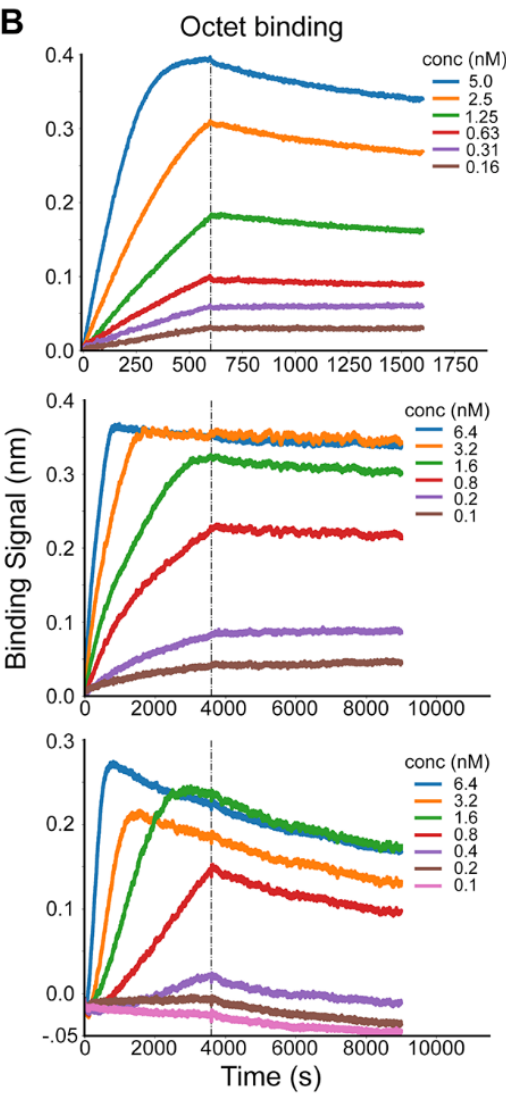

C

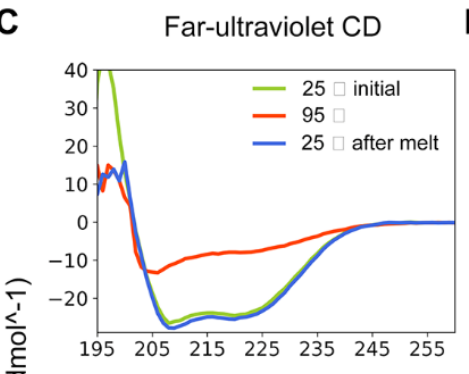

D Thermal Denaturation
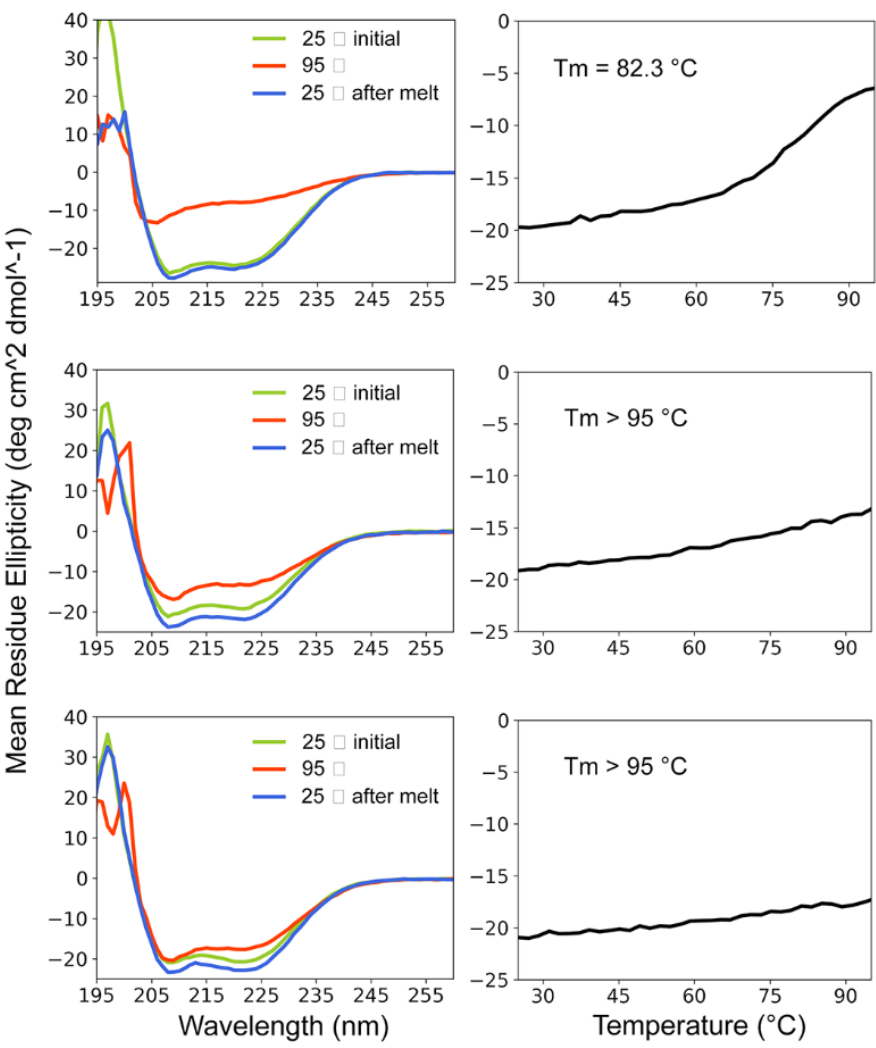

Fig. 3. The optimized designs bind with high affinity to the RBD, compete with ACE2, and are thermostable. (A) ACE2 competes with the designs for binding to the RBD. Yeast cells displaying the indicated design were incubated with 200pM RBD in the presence or absence of 1uM ACE2, and RBD binding to cells ( $Y$ axis) was monitored by flow cytometry. (B) Binding of purified miniproteins to the RBD monitored by biolayer interferometry. For LCB1 and LCB3 $K_{d}$ 's could not be accurately estimated due to lack of instrument sensitivity and long equilibration times below 200pM. (C) Circular dichroism spectra at different temperatures, and (D) CD signal at $222 \mathrm{~nm}$ wavelength as a function of temperature. The fully de novo designs LCB1 and LCB3 are more stable than the ACE2 scaffolded helix design AHB2. 
A

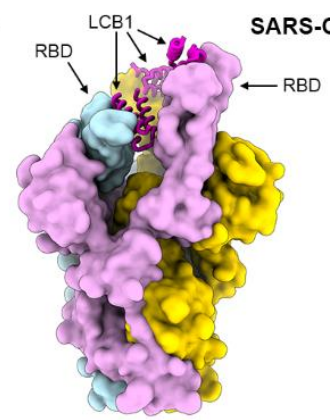

Viral membrane $=$

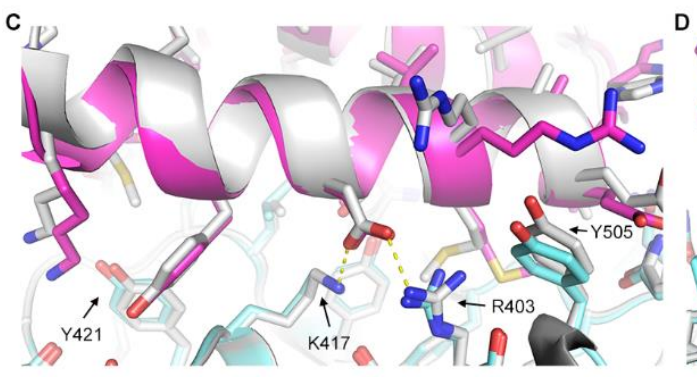

E

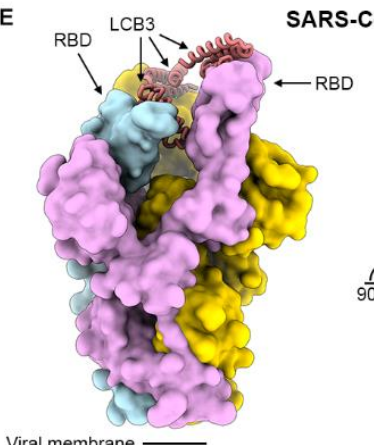

ARS-CoV-2 S bound to LCB3
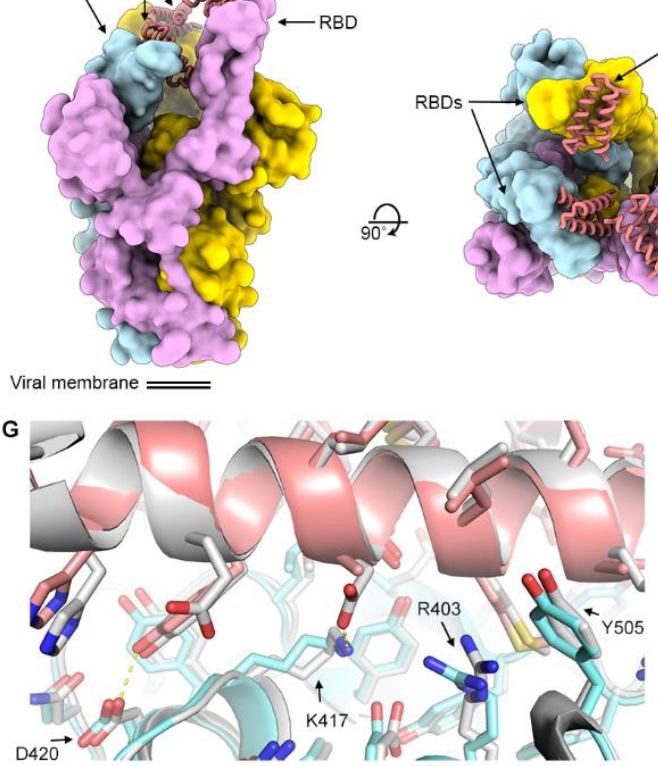

B

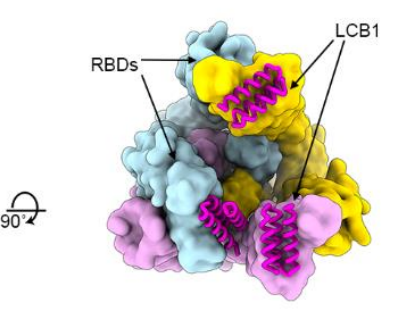

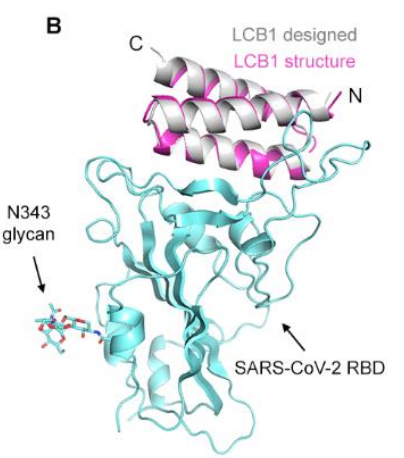
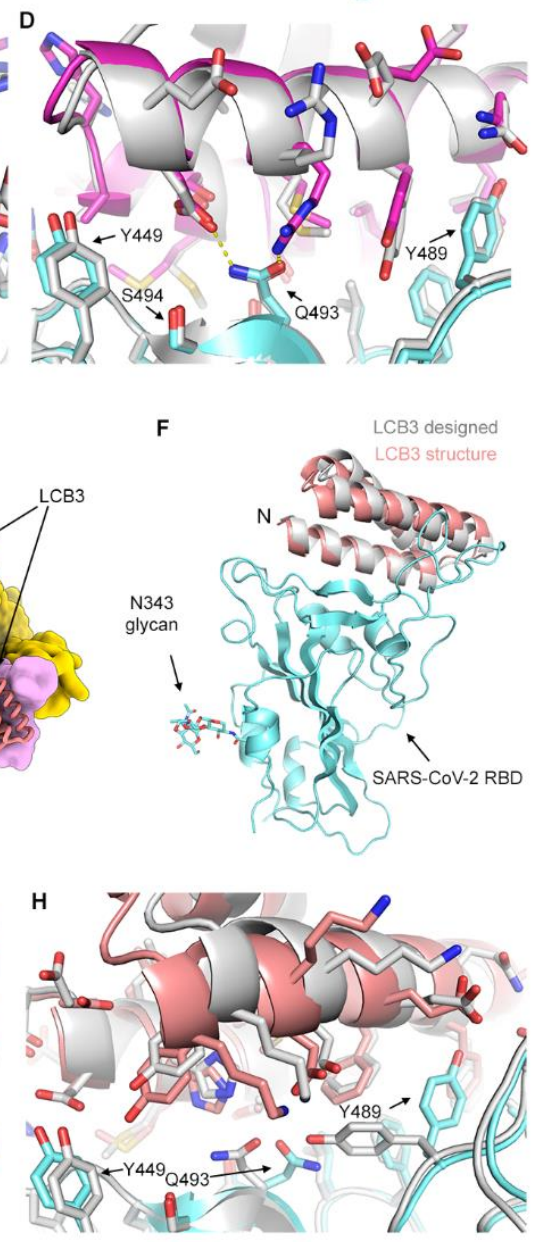

Fig. 4. CryoEM characterization of the LCB1 and LCB3 minibinders in complex with SARS-CoV-2 S. (A) Molecular surface representation of LCB1 bound to the SARS-CoV-2 S ectodomain trimer viewed along two orthogonal orientations.

Superimposition of the designed model (silver grey) and refined cryoEM structure (magenta) of LCB1 (using the map obtained through local refinement) bound to the RBD (cyan). (C and D) Zoomed-in views of computational model (silver grey) of LCB1/RBD complex overlaid on the CryoEM structure (cyan for RBD and pink for LCB1) showing selected interacting side chains. Molecular surface representation of LCB3 bound to the SARS-CoV-2 S ectodomain trimer viewed along two orthogonal orientations.

Superimposition of the designed model (silver grey) and refined cryoEM structure (salmon) of LCB3 (using the map obtained through local refinement) bound to the RBD (cyan). ( $\mathrm{G}$ and $\mathrm{H}$ ) Zoomed-in view of the interactions between LCB3 (salmon) and the SARS-CoV-2 RBD (cyan) showing selected interacting side chains. In (A) and (E), each $S$ protomer is colored distinctly (cyan, pink and gold). For (B) and (F), the RBDs were superimposed to evaluate the binding pose deviations between designed models and refined structure of each minibinder. 

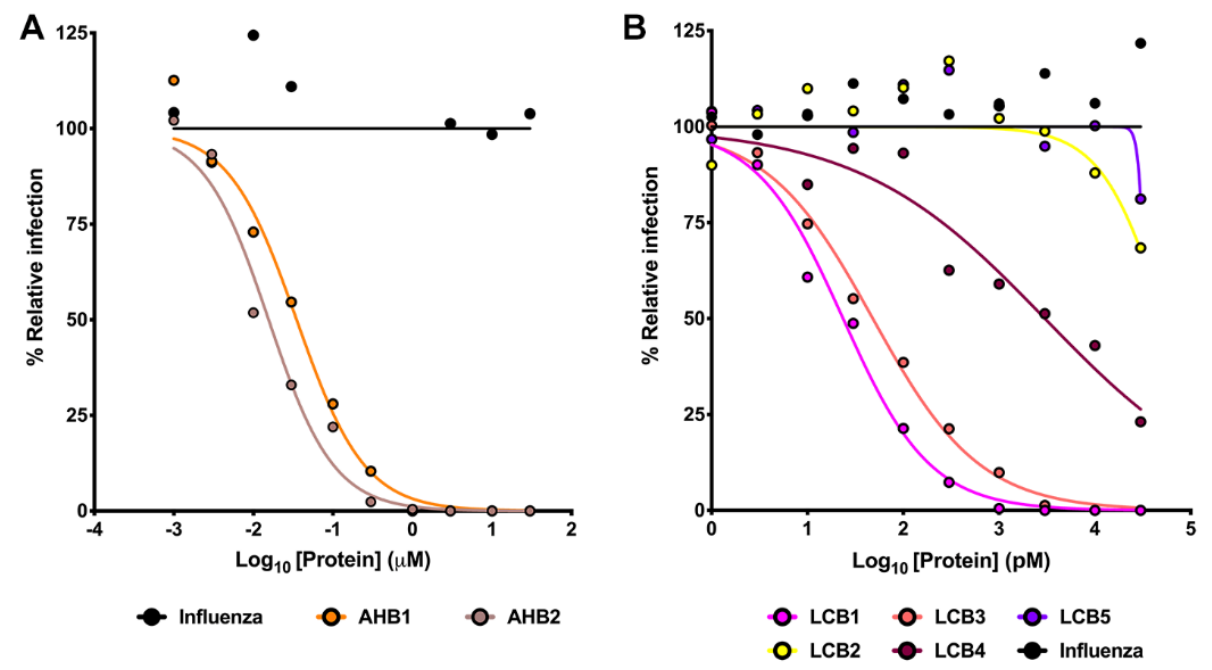

Fig. 5. Neutralization of live virus by designed miniprotein inhibitors. Neutralization activity of (A) AHB1 and AHB2 or (B) LCB1-5 were measured by FRNT. Indicated concentrations of minibinders were incubated with 100 FFU of authentic SARS-CoV-2 and subsequently transferred onto Vero E6 monolayers. AHB1, AHB2, LCB1, and LCB3 potently neutralize SARSCoV-2, with $\mathrm{EC}_{50}$ values $<50 \mathrm{nM}$ (AHB1 and AHB2) or < 50pM (LCB1 and LCB3). Data are representative of two independent experiments, each performed in technical duplicate. 